\title{
Case Report-Right iliac fossa mass in an HIV-positive woman
}

\author{
Kumwenda $J^{1}$, Nyirenda $M^{1}$ Tomoka $T^{2}$, \\ Ricken $D^{3}$, Chalulu K ${ }^{4}$, Kamiza $\mathbf{S}^{2}$ \\ 1. Department of Internal Medicine, College of Medicine, University of \\ Malawi. \\ 2. Department of Histopathology, College of Medicine University of \\ Malawi. \\ 3. Department of Obstetrics and Gynaecology, College of Medicine, \\ University of Malawi. \\ 4. Department of Surgery, College of Medicine, University of Malawi. \\ Corresponding author: JJ Kumwenda Email: jkumwenda@medcol.mw
}

A 35 year old woman was admitted to Queen Elizabeth Central Hospital (QECH) with fever, abdominal pain and vomiting for 3 days. A week earlier she had started antiretroviral therapy (ART) in a clinical trial in Blantyre, Malawi, after meeting all the requirements of the study. At the time of enrolment, her CD4 count was $19 / \mu$, and viral load $>400,000$ copies $/ \mathrm{ml}$. She was started on a combination of amtricitabine (FTC), tenofivir (TDF) and atazanavir (ATV).

At the present admission, she looked ill. Her axillary temperature was $38^{\circ} \mathrm{C}$, blood pressure $110 / 70 \mathrm{mmHg}$, pulse rate $100 /$ minute, weight $56 \mathrm{Kg}$. The abdomen was tender to touch without any palpable enlarged organs. A full blood count showed a haemoglobin concentration of $9.8 \mathrm{~g} / \mathrm{dl}$, other parameters being within normal limits. Blood biochemistry and liver function tests were normal. A blood sample for culture was taken which remained sterile after 7 days of incubation. The patient was started on empirical intravenous broad spectrum antibiotic (ceftriaxone $2 \mathrm{~g}$ once a day) for possible sepsis.

Two days later the patient remained ill and examination now revealed a right iliac fossa mass which was tender to touch. A bimanual pelvic examination suggested that the mass was arising from the pelvis. A gynecological review confirmed the findings. An ultrasound of the abdomen and pelvis identified a mass arising from the pelvis but was unable to define the organ of origin. Intravenous metronidazole was added to ceftriaxone. After ten days of antibiotic treatment, there was no clinical response.

The gynecologists decided to take the patient for surgical exploration. During the procedure a mass was found affecting the ileocaecal junction and the whole of the ascending colon. The visual findings were suggestive of a carcinoma of the ascending colon with areas of perforation. A hemi-colectomy was done (see figure 1-gross specimen of the colon)

The patient remained unwell and died two weeks later in hospital after repeated breakdown of the anastomosis site. A histology report came out two days after the patient died.

\section{What is the diagnosis?}

Figure 1: Part of the resected tumor
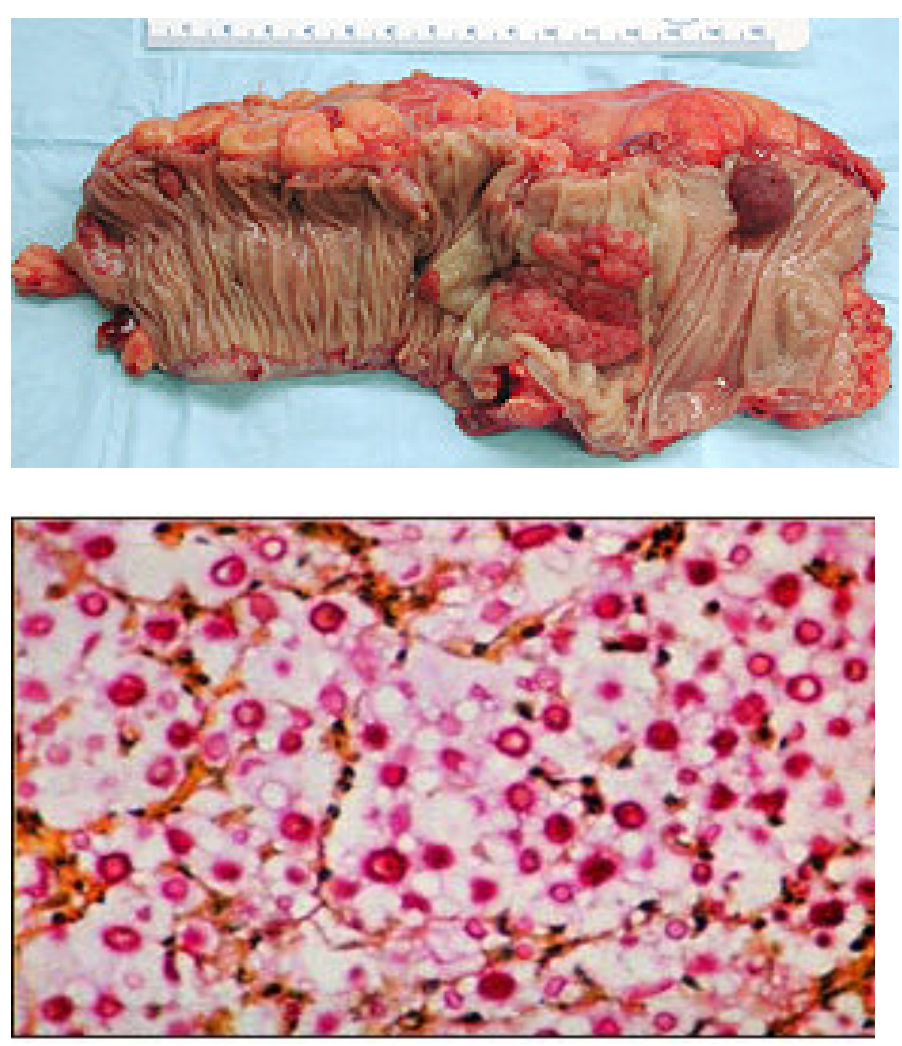

Figure 2: Histology Specimen stained with Periodic Acid Schiff reagent (PAS).

\section{Right iliac fossa mass in an HIV-positive woman}

Periodic Acid Schiff reagent (PAS), the stain used on the section shown in Figure 2, reacts with large carbohydrate molecules such as glycogen and polysaccharides, to produce a deep red or purple colour. It therefore delineates, for example, the surface structure of encapsulated microorganisms. In Figure 2, numerous distinct oval bodies can be seen throughout the sheet of tiussue. These are characteristic of the yeast Cryptococcus neoformans.

C neoformans can be isolated from soil and bird habitats. Most human infections are thought to be acquired by inhalation of fungus4. Pulmonary infection is usually asymptomatic. The organisms spread through the blood system and mainly cause meningoencephalitis, but can also affect other sites $^{5}$. Cryptococcosis due to C. neoformans is a common complication of severe immunodepression secondary to HIV infection ${ }^{6}$. Other conditions that predispose to cryptococcal infection include solid-organ transplantation or glucocorticoid therapy ${ }^{4}$. All these conditions share one common element, defective cell mediated immunity ${ }^{2}$.

The most common clinical presentation of cryptococcosis is meningitis, seen in over $70 \%$ of infections. About 6 to $10 \%$ of HIV infected persons not on prophylactic therapy or highly active antiretroviral therapy have been shown to develop cryptococcal meningitis in developed nations. More than $75 \%$ of the patients have CD 4 cell count that is less than $50 / \mu L 1,2$. Most infections are acquired through the respiratory tract, where the major host defense mechanism is complement-mediated phagocytosis by macrophages ${ }^{3}$. In about a third to half of cryptococcal infections, there is a 
pneumonitis. Pulmonary involvement is usually seen with dissemination, though isolated pulmonary disease may be present. Fever, cough, and dyspneoa are non-specific manifestations of pulmonary cryptococcosis. Other clinically apparent lesions may be seen on skin and in the eye $e^{4}$.

Cryptococcal meningitis is the commonest cause of adult meningitis in Malawians infected with HIV-1 contributing almost $60 \%$ of all proven adult meningitis cases at QECH (W'TL unpublished data). Intra-abdominal cryptococcosis however is rare.

This case is exceptional, not because a patient with severe HIV immunodepression developed cryptococcal infection but because the patient developed what looked like colonic carcinoma. There have been very few case reports to date of AIDS patients developing abdominal cryptococossis ${ }^{7,8}$ and even fewer case reports in HIV uninfected individuals. ${ }^{9}$. Our case is among the few and we believe it contributes to the body of knowledge in this area.

Other unusual predisposing factors to mesenteric cryptococcosis include $\mathrm{SLE}^{10}$. There has been one report of immuno-competent children developing mesenteric cryptococcosis ${ }^{11}$.

The development of a sudden right iliac fossa mass on the background of 10 days of HAART is difficult to explain. The mass developed too soon after starting ART to be considered an immune reconstitution syndrome. The mass may have been present earlier and missed during the original examination. Furthermore, a CD4 cell count performed two weeks after starting ART (at this point the patient was already admitted to the hospital) remained unchanged. If CD4 count had increased above baseline, immune reconstitution would be a possible explanation.

The host defense mechanisms protecting the body against cryptococcal infections are complex and not completely understood. Cell-mediated immunity (CMI) is the crucial component of the immune system for resistance against cryptococcosis, and its impairment presumably accounts for the high incidence of invasive cryptococcal infections in patients with HIV-AIDS. Other impairments of host defence may also contribute to susceptibility to crypotococcal invasion, as in a female SLE patient described by Mok et al, who developed cryptococcal meningitis concurrently with active SLE and in the absence of immunosuppressive treatment or other predisposing conditions ${ }^{12}$. They suggested that her low complement level, low natural killer cell count and the probable defective CD4 positive T cell and macrophage functions resulting from her SLE disease activity might have been responsible for her susceptibility to cryptococcal meningitis ${ }^{12,13,14}$.

\section{Conclusion.}

This 35 year old woman with severe HIV immunodepression presented with fever, abdominal pain, colonic inflammation and mesenteric lymphadenopathy which turned out to be due to colonic cryptococcosis. This is not a common diagnosis even in HIV infected individuals. Tuberculosis comes to mind more readily. We would like to suggest that Cryptococcus neoformans infection should be considered in the differential diagnosis of fever and marked abdominal lymphadenopathy in patients with AIDS. Where diagnostic facilities are limited empirical administration of antifungal agents may be necessary.

\section{References}

1.Minamoto GY, Rosenberg AS. Fungal infections in patients with acquired immunodeficiency syndrome. Med Clin North Am. 1997;81:389-409.

2.Chuck SL, Sande MA. Infections with Cryptococcus neoformans in the acquired immunodeficiency syndrome. $\mathrm{N}$ Engl $\mathrm{J}$ Med 1989;321(12):794-9

3.Miller GP. The immunology of cryptococcal disease. Semin Respir Infec 1986; 1: 45-52.

4.Bennet JE. Cryptococcosis. In: Braunwald E, Fauci AS, Kasper DL, Hauser SL, Longo DL, James JL, editors, Harrison's principles of internal medicine. 15th ed. New York: McGraw-Hill, 2001; 1174-5.

5.Lewis JL, Rabinovich S. The wide spectrum of cryptococcal infections. Am J Med 1972; 53: 315-22.

6.Eng RH, Bishburg E, Smith SM, Kapila R. Cryptococcal infections in patients with acquired immune deficiency syndrome. Am J Med 1986; 81: 19-23.

7.Scalfano FP Jr, Prichard JG, Lamki N, Athey PA, Graces RC. Abdominal cryptococcoma in AIDS: a case report. J Comput Tomogr 1988; $12: 237-9$.

8.Chong PY, Panabokke RG, Chew KH. Omental cryptococcoma. An unusual presentation of cryptococcosis. Arch Pathol Lab Med 1986; 110: $239-41$.

9.Daly, JS, ,Porter KA, Chong FK, Robillard RJ.: Disseminated, nonmeningeal gastrointestinal cryptococcal infection in an HIV-negative patient. Am J Gastroenterol. 1990 Oct;85(10):1421-4.

10.Kang IS, Park SH. Infectious complications in SLE after immunosuppressive therapies. Curr Opin Rheumatol 2003; 15: 52834.

11.Zou CC, Yu ZS, Tang LF, Liang L, Zhao ZY: Primary abdominal lymphonodular cryptococcosis in children: 2 case reports and a literature review. 1: J Pediatr Surg. 2006 Mar;41(3):11-

12.Mok CC, Lau CS, Yuen KY. Cryptococcal meningitis presenting concurrently with systemic lupus erythematosus. Clin Exp Rheumatol 1998; 16: 169-71.

13.Abu-Shakra M, Urowitz MB, Gladman DD, Gough J. Mortality studies in systemic lupus erythematosus. Results from a single center. II. Predictor variables for mortality. J Rheumatol 1995; 22: 1265-70.

14.Tsokos GC, Kovacs B, Sfikakis PP, Theocharis S, Vogelgesang S, Via CS. Defective antigen-presenting cell function in patients with systemic lupus erythematosus. Arthritis Rheum 1996; 39: 600-9. 